\title{
JOGOS DIGITAIS PARA INTERAÇÃO COM BRAIN COMPUTER INTERFACE PARA AUXILIAR NO PROCESSO DE INCLUSÃO ESCOLAR DE PESSOAS COM PARALISIA CEREBRAL
}

Regina de O. Heidrich

Universidade Feevale rheidrich@feevale.br

Marsal A. Branco Universidade Feevale marsal@feevale.br

\author{
João B. Mossmann \\ Universidade Feevale \\ mossmann@feevale.br \\ Anderson R. Schuh \\ Universidade Feevale \\ andersonrs@feevale.br \\ Emely Jensen \\ Universidade Feevale \\ emelyjensen@gmail.com
}

Resumo: Uma Interface Cérebro-Computador $(\mathrm{BCl})$, permite que uma pessoa possa transferir comandos a um computador diretamente. Em vez de utilizar um teclado, mouse ou outro dispositivo de entrada, o utilizador desta interface simplesmente emite os comandos através de ondas cerebrais e o computador responde a eles. Este trabalho pretende apresentar um game desenvolvido para auxiliar o processo de inclusão escolar de pessoas com paralisia cerebral. Método: Pesquisa de abordagem qualitativa. Para o desenvolvimento desta pesquisa escolhemos o estudo de caso pois o mesmo é uma investigação multifacetada, em profundidade, de um único fenômeno social. Resultados: Desenvolvimento do Game Neuro-Navegática, com diferentes formas de velocidade de varredura, o software permite que o usuário através do piscar de olhos possa escolher a matéria. Neste exemplo optou-se pela matemática. Conclusão: $O$ estudo sobre Brain Computer Interface $(\mathrm{BCl})$ busca aprimorar a maneira de interação entre o ser humano e as máquinas e permite que pessoas com paralisia cerebral possam se beneficiar no processo de educação inclusive, assim como seus colegas sem deficiência.

Palavras-chave: Ergonomia cognitiva, Interação, Brain Computer Interface (BCl), Paralisia Cerebral, Games.

Abstract: Introduction: A Brain-Computer Interface (BCI), allows a person to transfer commands to a computer directly. Instead of using a keyboard, mouse or other input device, the user of this interface simply sends commands via brain waves and the computer responds to them. This paper aims to present a game developed to assist the process of educational inclusion of people with cerebral palsy. Method: Qualitative research approach. To develop this research, we chose the case study, because it is a 
multifaceted research, in depth, of a single social phenomenon. Results: Development of the Game Neuro-Navegática, with different forms of scanning speed, the software allows the user through the blink of an eye to choose the subject. In this example we opted for mathematics. Conclusion: The study on Brain Computer Interface (BCl) seeks to improve the way of interaction between humans and machines and allows people with cerebral palsy to may be benefited in the inclusive education process as well as their peers without disabilities.

Keywords: Cognitive Ergonomics, Interaction, Brain Computer Interface $(B C I)$, Cerebral Palsy, Games.

\section{INTRODUÇÃO}

Uma interface cérebro-computador $(\mathrm{BCl})$, permite que uma pessoa possa transferir comandos a um computador diretamente. Em vez de utilizar um teclado, mouse ou outro dispositivo de entrada, o utilizador desta interface simplesmente emite os comandos através de ondas cerebrais e o computador responde a eles. 0 processo de inclusão escolar tem sido amplamente discutido em todas as esferas e têm-se observado que por trás do discurso dos professores de não sentirem-se preparados, a falta de acessibilidade e formação na área de inclusão digital é ainda muito incipiente. Uma das soluções é a parceria com grupos e projetos de pesquisa de universidades que poderão colaborar neste processo. A inclusão de pessoas com paralisia cerebral não é fácil, pois muitos têm o cognitivo preservado mas não falam, caminham, ou ambos. Pretende-se mostrar aqui, uma solução de objetos de aprendizagem desenvolvidos com a utilização da tecnologia Brain Computer Interface $(\mathrm{BCl})$.

Pessoas com paralisia cerebral, que tem o cognitivo preservado, mas não podem se comunicar, locomover, ou ambos, necessitam de um auxilio tecnológico para sua aprendizagem. A utilização de objetos de aprendizagem poderá envolver conceitos e conhecimentos através de práticas de experimentação que não seriam possíveis de outras formas. Dessa maneira poderemos simular situações educacionais facilitando o processo de inclusão escolar destas pessoas. Assim fica evidente que novas técnicas devem ser concebidas dentro de diretrizes de acessibilidade, a fim de garantir ambientes digitais mais inclusivos. O termo objeto educacional (learning object) geralmente aplica-se a materiais educacionais projetados e construídos em pequenos conjuntos com vistas a maximizar as situações de aprendizagem onde 0 recurso pode ser utilizado. A ideia básica é a de que os objetos sejam como blocos com os quais será construído o contexto de aprendizagem. O IEEE Learning Technology Standards Committee (2002, p.5) vai mais além, segundo esta instituição "Qualquer entidade, digital ou não digital, que possa ser usada, reutilizada ou referenciada durante o uso de tecnologias que suportem ensino". Um ambiente em objetos de aprendizagem é um software que tem um objetivo, onde o aluno realiza decisões que interagem com as ações próprias do ambiente de interação e resultam em uma nova condição. Dessa forma, as novas propostas de ensino buscam proporcionar cenários de aprendizagem que estimulem o desenvolvimento da autonomia intelectual e isto é possível por meio de processos de aprendizagem que promovem o raciocínio e a 
revolução de problemas de forma coletiva (BARROS, 1994). Objetos de aprendizagem em forma de Construtos Digitais de Aprendizagem (CDAs) apresentam-se como uma tecnologia assistiva que oferece prosperas contribuições ao desenvolvimento de aplicações voltadas para o ensino. "Para as pessoas sem deficiência a tecnologia torna as coisas mais fáceis. Para as pessoas com deficiência, a tecnologia torna as coisas possíveis" (RADABAUGH, 1993). Já Cook e Hussey definem a TA citando o conceito do ADA - American with Disabilities Act, como "uma ampla gama de equipamentos, serviços, estratégias e práticas concebidas e aplicadas para minorar os problemas funcionais encontrados pelos indivíduos com deficiências" (COOK \& HUSSEY, 1995). O Comitê de Ajudas Técnicas - CAT ${ }^{1}$ do ministério da Ciência e Tecnologia do Brasil aprovou e reconheceu, em 14 de dezembro de 2007, este novo conceito:

Tecnologias Assistivas. É uma área do conhecimento, de característica interdisciplinar, que engloba produtos, recursos, metodologias, estratégias, práticas e serviços que objetivam promover a funcionalidade, relacionada a atividade e participação de pessoas com deficiência, incapacidades ou mobilidade reduzida, visando sua autonomia, independência, qualidade de vida e inclusão social.

O conceito de Tecnologia assistiva é considerado amplo, podendo ser peça chave na promoção dos Direitos Humanos, pois possibilita a pessoa com deficiência a oportunidade de alcançar autonomia e independência em diversos aspectos da sua vida (SECRETARUA DE DUREITOS HUMANOS DA PRESIDÊNCIA DA REPUBLICA, 2009). Deficiência é a ausência ou difusão de uma estrutura psíquica, fisiológica ou anatômica. Diz respeito à biologia da pessoa. A deficiência física causa uma desvantagem, resultante de um comprometimento ou de uma incapacidade, que limita ou impede o desempenho motor de determinada pessoa (Fernandes et al., 2007).

Um CDA segundo Branco et al. (2013), qualquer entidade ou artefato inventado ou construído de maneira multidisciplinar no formato de um game educacional, ajudando os jogadores a construir ou reelaborar seu conhecimento. Assim, têm como características a natureza dual, como jogo e objeto de aprendizagem e também jogo, por trabalhar processualmente as contribuições de cada ara sem nenhum tipo de subordinação entre elas. Assim, a partir do $\mathrm{BCl}$, o utilizador não usa mouse, mas um hardware específico onde comanda o computador através de ondas cerebrais.

Dentre as tecnologias assistivas aplicáveis no contexto desse trabalho, destacam-se os jogos digitais, que atualmente também passam a ser considerados uma forma de linguagem, visto que podem transformar conteúdos tradicionais em novos conteúdos interativos e comunica-los de uma maneira mais eficiente e inovadora.

Este é um conceito novo, o qual é chamado de gamification, ele permite criar simulações, com o objetivo de capacitar profissionais, bem como o auxílio ao aprendizado de alunos. Segundo Zichermann e Cunningham (2011), todos estão incorretos, e assim definem Gamificação como sendo a utilização de características de

\footnotetext{
${ }^{1}$ Disponível na internet por http em: $<$ http://www.pessoacomdeficiencia.gov.br/app/sites/default/files/publicacoes/livro-tecnologiaassistiva.pdf>
} 
jogos, como pensamentos e mecânicas, como forma de envolver usuários na busca por algum objetivo ou resolução de algum problema. A gamificação, como conceito, tem sido sistematicamente mal interpretada; é errado pensar que se trada de uma ciência que se debruça sobre o ato de criar jogos, mas sim uma metodologia por meio da qual se aplica mecanismos de jogos à resolução de problemas ou impasses em outros contextos (ZICHERMANN; CUNNIGHAM, 2011, p.17).

\subsection{Paralisia Cerebral}

Qualquer movimento humano, desde o mais simples, requer um sistema complexo de comunicação que envolve o cérebro, nervos e músculos. Quando uma área do sistema nervoso que regula o movimento apresenta uma lesão ou uma anormalidade, o indivíduo pode apresentar uma ampla variedade de distúrbios do movimento. A paralisia cerebral (PC) ou encefalopatia não progressiva crônica da infância (ECNPI) é um distúrbio da postura e do movimento, resultante de uma lesão ou encéfalo imaturo nos períodos pré, peri ou pós-natal (FINNIE, 2000; SCHWARTZMAN, 2004; SANKAR; MUNDKUR, 2005; GERALIS, 2007). Em 2006, o Executive Committee for the Definition of Cerebral Palsy formulou uma definição que descreve a paralisia cerebral como um grupo de desordens permanentes do desenvolvimento da postura e do movimento, atribuídas a perturbações não progressivas ocorridas no desenvolvimento fetal ou infantil.

\subsection{Características dos Games}

Todos os jogos compartilham quatro características que os definem: meta, regras, sistema de feedback e participação voluntária" (MCGONIGAL apud MEDINA et al., 2013, p 28). A meta é o motivo que justifica a realização de uma atividade por parte dos jogadores, em outras palavras, o elemento pelo qual os participantes de um jogo concentram suas atenções para atingir os propósitos designados. As regras ajustam o nível de complexidade do jogador frente à atividade a ser desenvolvida, liberando a criatividade e estimulando o pensamento estratégico [...] portanto, têm a função de definir a maneira pela qual o jogador se comportará, ou de que modo organizará suas ações para o cumprimento dos desafios impostos pelo jogo" (MEDINA et al., 2013, p. 28). O sistema de feedback, informa aos jogadores como está sua relação com os diferentes aspectos que regulam sua interação com a atividade. Também cabe a esse sistema fomentar motivação, mantendo os participantes constantemente conscientes do progresso atingido em relação a si próprios e à meta. (Medina et al., 2013, p. 28-29). Observa-se segundo o mesmo autor que em qualquer tipo de jogo, digital ou não, é preciso haver consenso entre todas as condições propostas e o jogador.

Apesar de haver poucas pesquisas, Granic, Adam e Rutger (2013) apontam sobre os benefícios de jogar vídeo game, especificamente, as funções e os benefícios do jogo de forma mais geral têm sido estudados há décadas. A psicologia evolucionista tem enfatizado há muito tempo as funções adaptativas de jogo (BJORKLUND; PELLEGRINI, 2010), e da psicologia do desenvolvimento, a função positiva de jogo tem 
sido um tema recorrente para alguns dos mais respeitados estudiosos da área (por exemplo, Erikson, 1977; Piaget, 1962; Vygotsky, 1978). Erikson (1977) propôs que os contextos de jogos permitem que as crianças a experimentem com as experiências sociais e simulem consequências emocionais alternativas, que podem trazer sentimentos de resolução fora do contexto de jogo. Da mesma forma, Piaget (1962) teorizou que o jogo de faz de conta oferece às crianças oportunidades para reproduzir os conflitos da vida real, para elaborar resoluções ideais para seu próprio prazer, e para amenizar sentimentos negativos. Tanto Piaget (1962) e Vygotsky (1978) defendiam fortes ligações teóricas entre jogo e uma variedade de elementos que favorecem o desenvolvimento da cognição social.

Em suma, tipos específicos de jogos de vídeo game parecem reforçar um conjunto de funções cognitivas, alguns dos quais parecem simular contextos do mundo real e auxiliar o desenvolvimento de processos cognitivos. Estes dados sugerem que as ordens para proibir jogos violentos podem ser demasiado simplistas. No mínimo, a pesquisa sobre o impacto negativo destes jogos precisa ser equilibrada com as evidências para os benefícios cognitivos desses mesmos jogos.

Passamos agora para os benefícios motivacionais, emocionais e sociais de jogar vídeo games. No ponto de vista da educação, Johnson (2005) defende que, não importa o que o jogador pensa enquanto joga. O que importa é a maneira como o jogador está pensando. Essa afirmação é reforçada por Dewey (1997) ao defender que a maior de todas as falácias pedagógicas talvez seja a noção de que uma pessoa aprende apenas aquela determinada coisa que está estudando. Nesse aspecto, destaca a aprendizagem colateral no caminho para formar atitudes de construção duradouras, sendo essas, com frequência, mais importantes do que as aulas de gramática ou as aulas de geografia e história que são aprendidas. Assim, ao jogar os usuários criam construções, a partir do aprendizado colateral. Há algum tempo, empresas vem empenhando seus esforços para desenvolver dispositivos de baixo custo, a fim de possibilitar o uso em nível pessoal. Neste trabalho os games e o $\mathrm{BCl}$ também podem ser denominados como um tipo de tecnologia assistiva.

\subsection{Brain computer interface $-\mathrm{BCl}$}

Wolpaw (2007) afirma que as Interfaces cérebro-computador (BCl) são fundamentalmente uma nova abordagem para restaurar a comunicação e controle para pessoas com distúrbios motores graves, como Esclerose Lateral Amiotrófica (ELA) e outras lesões medulares e doenças degenerativas. E afirmou, em 2007, que poderia ser uma excelente tecnologia assistiva.

Schuh et al. (2013), desenvolveu um estudo e prototipação de um simulador de cadeira de rodas em ambiente tridimensional controlado por interface cérebro computador não-invasivo. Para tal, foi utilizado um EEG de baixo custo, o NeuroSky Mindwave (MW), como dispositivo de aquisição de sinais. Para desenvolvimento, foi utilizado o Unity3 $\mathrm{D}^{2}$, um motor de jogos. Através do protótipo desenvolvido foi

$2 \quad A$ Unity3D é um motor de jogos (do inglês Game Engine) para desenvolvimento em ambientes 2D e 3D. A ferramenta veio ao mercado com o intuito de democratizar o desenvolvimento de jogos, assim possui atualmente 1,5milhoes de usuários registrados, dentre eles desenvolvedores amadores até 
possível detectar o piscar dos olhos, e assim, utilizar esta característica como comando para o simulador.

Parasuraman e Rizzo (2008) da Universidade George Mason durante a Army Science Conferece que decorreu em Orlando 2008, apresentou um estudo profundo que revela a importância da análise das funções cerebrais com o objetivo de aumentar a performance humana em sistemas complexos. No livro Neuroergonomics (Oxford University Press, 2008), apresentam-se alguns entendimentos necessários para a concepção efetiva de sistemas homem-máquina. O autor afirma que para essa concepção é necessário compreender a mente humana em relação ao trabalho e à tecnologia, e que isso é papel da Ergonomia. A Ergonomia Cognitiva é um ramo da ergonomia. É um conjunto de fatores que incide sobre o ajuste entre as habilidades e limitações cognitivas humanas entre máquinas, tarefas e meio ambiente. Guimarães (2004) afirma que a Ergonomia Cognitiva é definida como a área que "engloba os processos perceptivo, mental e de motricidade". Abbad e Borges-Andrade (2004) apontam que a preocupação da Ergonomia Cognitiva está voltada aos seguintes processos mentais:

a) Domínio Cognitivo;

- conhecimento, compreensão, aplicação, análise, síntese e a avaliação;

b) Domínio Afetivo;

- receptividade, resposta, valorização, organização e caracterização;

c) Domínio Psicomotor;

- percepção, posicionamento, mecanização e domínio completo.

A mente humana não pode ser compreendida sem um estudo do cérebro, e que isto é competência das neurociências, daqui resulta que o estudo do cérebro e da mente no domínio complexo do trabalho só é possível através da Neuroergonomics. A Neuroergonomics pode assim fornecer interações mais eficazes e naturais entre 0 homem e a tecnologia.

\subsection{Funcionamento}

Conforme a Figura 1 apresenta, pode-se observar:

a) O processo começa na intenção do usuário;

b) A intenção de comunicação ou controle de algo desencadeia um processo complexo em certas áreas do cérebro;

c) A ativação de certas áreas do cérebro provoca uma diferença de potencial com as áreas adjacentes. 


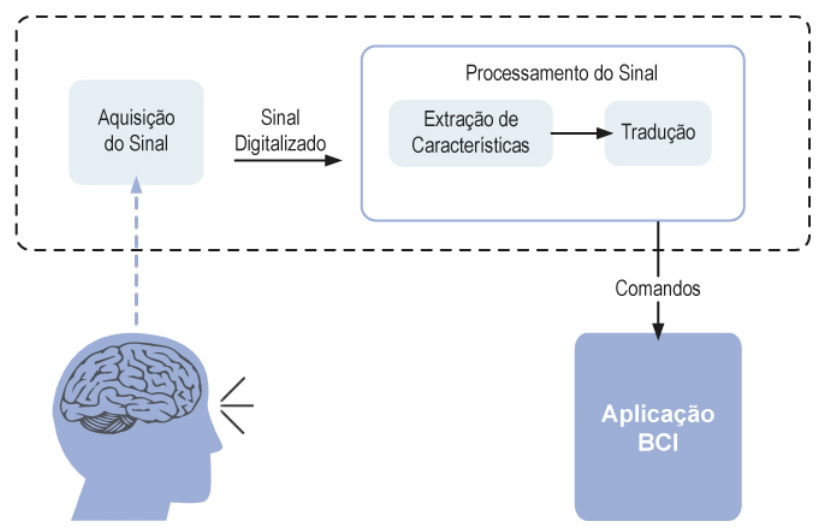

Figura 1 - Diagrama $\mathrm{BCl}$

Fonte: $\mathrm{O}$ autor

O hardware utilizado neste projeto é denominado Mindwave desenvolvido pela empresa NeuroSky, e funciona como BCl (Brain Computer Interface). Tem como objetivo: utilizar o cérebro como interface de comunicação com a máquina. Ele converte ondas cerebrais elétricas e processos analógicos em sinais digitais para fazer medições disponíveis para alimentar a interface de usuário de jogos, computadores e aplicações médicas de pesquisa. É fácil de usar, não invasivo, sensor único seco, permite mobilidade, o acesso a ambos os dados brutos ou dados é feito através de algoritmos optimizados e a plataforma aberta para qualquer indústria.

Cada interação entre os neurônios cria uma descarga elétrica minúscula, mensurável por máquinas de EEG (eletroencefalograma). Por si só, estas acusações são impossíveis de medir do lado de fora do crânio. No entanto, um estado mental dominante, impulsionado pela atividade dos neurônios coletivamente criado por centenas de milhares de descargas simultâneas, pode ser medido.

\subsection{Objetivos}

A seguir os objetivos do artigo:

a) Apresentar as possibilidades de utilização de games na educação;

b) Demonstrar a utilização de Brain Computer interface no desenvolvimento de games;

c) Apresentar algumas telas do game envolvido, onde a interface de interação é o piscar dos olhos.

Neste estudo apresenta-se o uso de games e a associação do $\mathrm{BCl}$, como uma tecnologia assistiva, a partir do uso de eletroencefalograma que por muito tempo foi restrito a área medica e laboratórios de pesquisa.

\subsection{Materiais e Métodos}

Pesquisa de abordagem qualitativa. Para o desenvolvimento desta pesquisa escolhemos o estudo de caso pois o mesmo é uma investigação multifacetada, em profundidade, de um único fenômeno social. É conduzido em grande detalhe e, com frequência, se baseia no uso de várias fontes de dados. (FEAGIN; ORUM; SJOBERG, 1991). Bogdan e Biklen (1982) definem algumas categorias dentro do estudo de caso. A 
categoria que iremos trabalhar é a de Estudos de Casos Observacionais. Conforme este autor, pode ser objeto da observação participante o trabalho realizado dentro da sala de aula e novos métodos de ensino. Stake (1995) sugere que o estudo de caso é o estudo da particularidade e complexidade de um caso para entender suas atividades dentro de circunstâncias especiais. Conforme Patton (1987), o estudo de caso procura detalhar e descrever a unidade em profundidade, explorando-a de forma holística. Esta análise precisa ser feita de forma cuidadosa, pois a observação é o principal elemento na sua coleta de dados. Está pesquisa trata de um múltiplo estudo de caso exploratório descritivo (YIN, 1989). Além disso, consultar as necessidades e sugestões dos usuários através de duas maneiras, considerando a metodologia do design social: método de projeção que considera como precedentes e como indutores as opiniões dos usuários, bem como que compartilha com os usuários os estudos e as definições posteriores, de modo que os usuários sintam-se participes do processo e do resultado. As etapas deste estudo são:

a) Estudo das tecnologias em Jogos Digitais;

b) Verificar como uma pessoa com restrições motoras interface com os jogos, bem como os hardwares específicos;

c) Desenvolver a modelagem;

d) Desenvolver o protótipo;

e) Testar a tecnologia com os usuários;

f) Realizar a modelagem do game;

g) Desenvolver o protótipo a partir de novos testes;

h) Testar a interação dos usuários com o game;

Desta maneira pretende-se levar para as escolas públicas da região, possibilidades de interação entre pessoas com paralisia com pessoas sem paralisia.

\subsection{Resultados}

A Figura 2 mostra um exemplo de figura. Citar a origem da informação. Se a origem for própria, apor "Elaborado pelo autor, com base na pesquisa realizada"

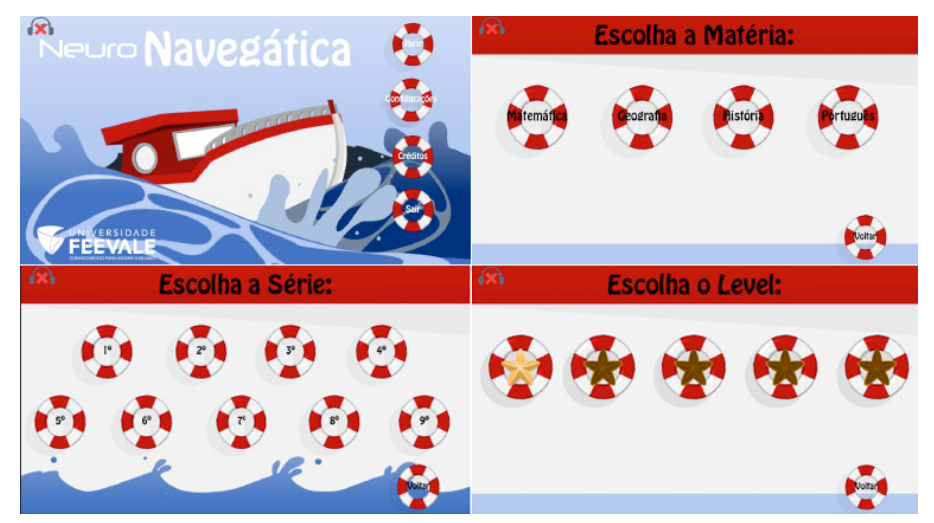

Figura 2 - Telas iniciais do jogo Neuro-Navegática.

Fonte: Adaptado do jogo Neuro-Navegática. 
Aqui o usuário poderá selecionar a série de acordo com a sua escolarização e o nível, que funciona como as frases do game. No exemplo apresentado na Figura 3, selecionou-se a 3a série.

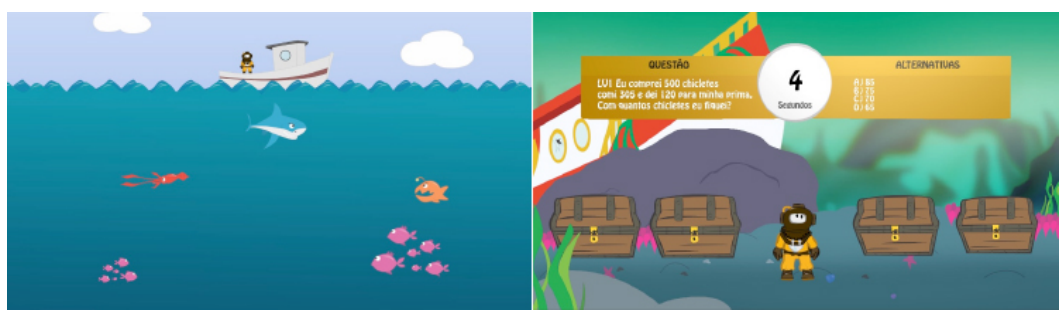

Figura 3 - Tela do jogo Neuro-Navegática.

Fonte: Adaptado do jogo Neuro-Navegática.

Ao final o mergulhados seleciona, através da opção de varredura na tela, com o piscar de olhos a resposta correta.

\section{CONCLUSÃO}

A tecnologia $\mathrm{BCl}$ (Brain Computer Interface) proporciona uma forma de interação com maquinas, produtos, sistema e como tal, revela-se com grande importância o seu estudo, pois por um lado, através do $\mathrm{BCl}$ já é possível a adaptação de maquinas, produtos, sistemas a população com problemas de mobilidade para melhorar o seu desempenho, transformando incapacidades em meras diferenças de execução, mas com medias de desempenho semelhantes as pessoas comuns. Por outro lado o estudo do $\mathrm{BCl}$ para a ergonomia permitirá analisar níveis de carga mental de forma instantânea e objetiva. Uma interface Cérebro-Computador (ICC) é um sistema computacional capaz de estabelecer a comunicação entre a atividade neurofisiológica e um computador. O estudo sobre ICC busca aprimorar a maneira de interação entre o ser humano e as maquinas. É importante lembrar que a ampliação e recuperação das funções motora e cognitiva, são o principal foco das pesquisas desta área. Pode-se afirmar que o EEG, apesar de ter sido desenvolvido há bastante tempo, ainda é uma ferramenta fundamental para o apoio a diagnósticos clínicos. Entretanto, pesquisadores estão realizando novas abordagens para este dispositivo, entre elas, estão as ICCs. Atualmente, existem no mercado dispositivos que aproximam o usuário final das ICCs. Em contrapartida, ainda hoje, não existem aplicações que façam essa interação de maneira convencional. E acredita-se que brevemente veremos tais utilizações aliadas aos games na educação inclusiva.

Na pós-modernidade, num momento que vários países buscam uma educação inclusiva, onde pessoas com deficiência possam conviver em diferentes ambientes educacionais, a possibilidade de usar games e dispositivo de $\mathrm{VCl}$, traz novas esperanças para esta camada da população. No Brasil $23,9 \%$ da população apresenta alguma deficiência segundo dados do último Censo de 2010 (SECRETARIA DE DIREITOS HUMANOS DA PRESIDÊNCIA DA PREPÚBLICA).

Podemos vislumbrar um campo de estudos com grandes possibilidades de sucesso na área escolar e também no mundo do trabalho. Também observamos que nos vários grupos de pesquisadores de universidades renomadas não houve nenhuma 
publicação que mostrasse a pesquisa realizada com pessoas com paralisia cerebral e BCS.

\section{REFERÊNCIAS}

ANDRADE, J. M. P. Paralisias Cerebrais. [ca.1999]. Disponível na internet por http em: <http://infoativodefnet.blogspot.com.br>. Acesso em 13 jun. 2014.

BARROS, L. A. (1994). Suporte a Ambientes Distribuídos para Aprendizagem

Cooperativa. 1994. Tese (Doutorado) - Universidade Federal do Rio de Janeiro, Rio de Janeiro, Curso de Pós-Graduação em Engenharia de Software.

BJORKLUND, D. F.; PELLEGRINI, A. D. Evolutionary perspectives on social development. In: SMITH, P. K.; HART, C. H. (Eds.), The Wiley-Blackwell handbook of childhood social development. Oxford: Wiley-Blackwell, 2010. p. 64-81.

BOGDAN, R.; BIKLEN, S. K. Qualitative research for education: an introduction for to theory and Methods. Boston: Allyn and Bacon, 1982.

BRANCO, M. A. et al. Dimensões dos jogos de ensino. ICECE 2013 - Congresso Internacional em Educação em Engenharia e Computação 2013. Anais do ICECE 2013, v. 8, p. 277-281, 2013. Disponível na internet por http em:

<http://proceedings.copec.org.br/index.php/icece/article/view/457>. Acesso em 11 jun. 2013.

COOK, A.M.; HUSSEY, S. M. Assistive Technologies: Principles and Practices. St. Louis: Mosby, 1995.

DEWEY, J. Experience and Education. New York: Free Press, 1997.

ERIKSON, E. H. Toys and reasons: Stages in the ritualization of experience. New York: Norton, 1977.

EXECUTIVE COMMITTEE FOR THE DEFINITION OF CEREBRAL PALSY. A report: the definition and classification of cerebral palsy April 2006. Developmental Medicine \& Child Neurology, v. 49, n. 109, p. 8-14, fev. 2007.

FEAGIN, J.; ORUM, A.; SJOBERG, G. A Case for the Case Study. Chapel Hill: The University of North Carolina Press, 1991.

FERNANDES, A. C. et al. Medicina e Reabilitação: princípios e prática. São Paulo: Artes Médicas, 2007.

FINNIE, N. R. O manuseio em casa da criança com paralisia cerebral. 3. ed. São Paulo: Manole, 2000.

GERALIS, E. Crianças com paralisia cerebral: guia para pais e educadores. 2. ed. Porto Alegre: Artmed, 2007.

GRANIC, I.; LOBEL, A.; ENGELS, R. C. M. E. The benefits of playing video games.

American Psychologist, n. 69, p. 66-78, 2014.

GUIMARÃES, L. B. M. Ergonomia Cognitiva: Produto e Produção. Porto Alegre: FEENG, 2004. 
JOHNSON, S. Surpreendente: a Televisão e o Videogame nos Tornam Mais Inteligentes. São Paulo: Campus, 2005.

MEDINA, B. et al. Gamification Inc.: como reinventar empresas a partir de jogos. 1. ed. Rio de Janeiro: MJV Press, 2013.

PATTON, M. Q. How to use qualitative Methods in Evaluation. London: Sage, 1987.

SANKAR, C.; MUNDKUR, N. Cerebral Palsy - Definition, Classification, Etiology and Early Diagnosis. The Indian Journal of Pediatrics, Nova Déli, v. 72, n. 10, p. 865-868. out. 2005.

SCHWARTZMAN, J. S. Paralisia cerebral. Arquivos Brasileiros de Paralisia Cerebral, v. 1, n. 1, p. 4-17, 2004.

SECRETARIA DE DIREITOS HUMANOS DA PRESIDÊNCIA DA REPÚBLICA. Cartilha do Censo 2010 - Pessoas com Deficiência. Brasília: SDH-PR/SNPD, 2012. Disponível na internet por http em:

$<$ www.pessoacomdeficiencia.gov.br/app/sites/default/files/publicacoes/cartilhacenso-2010-pessoas-com-deficienciareduzido.pdf>. Acesso em 13 jun. 2014.

SECRETARIA DE DIREITOS HUMANOS DA PRESIDÊNCIA DA REPÚBLICA. Tecnologia Assistiva. Brasília, DF: Comitê de Ajudas Técnicas, 2009.

STAKE, R. E. The Art of Case Study Research. London: Sage, 1995.

VYGOTSKY, L. Mind in society: The development of higher psychological functions. Cambridge: Harvard University Press, 1978.

WOLPAW, J. R. Brain-computer interfaces as new brain output pathways. The Journal of Physiology, v. 579, n. 3, 613-619, mar. 2007.

YIN, R. K. Case Study Research: Design and Methods. 2. ed. Thousand Oaks: Sage, 1994. 